\title{
Study on image segmentation in CT metal artifacts
}

\author{
Ziyue Yan ${ }^{1,2}$, Xuelong $\mathrm{Hu}^{2}$, Lifeng Zhang ${ }^{1}$, Seiichi Serikawa ${ }^{1}$ \\ ${ }^{1}$ Department of Electrical Engineering and Electronics, Kyushu Institute of Technology, 1-1 Sensui-cho,Tobata-ku, \\ Kitakyushu 804-8550,Japan \\ ${ }^{2}$ School of Information Engineering, Yangzhou University, 198 Huayangxi Road, Hanjiang, Yangzhou 225127, China \\ *Corresponding Author: yanziyue07@gmail.com
}

\begin{abstract}
Computed Tomography (CT) is one of the most important means of medical diagnosis and the quality of CT image can be seriously affected by metal artifacts. How to use CT image segmentation to extract the focused region is a classical difficult problem in this research field. According to the principle of CT reconstruction, after the medical image segmentation, projection of the metal part by compensation can improve the image quality. This paper first introduces the causes of the metal artifacts as well as the principle of CT image reconstruction. Then, it mainly discusses the simple and iterative threshold segmentation to solve metal artifacts. Corresponding comparison shows that the proposed method in this study has better segmentation effect based on the experimental results. Finally, the prospect of medical image segmentation is predicted to indicate future research work.
\end{abstract}

Keywords: metal artifacts, image segmentation, image quality, threshold segmentation.

\section{Introduction}

Nowadays, X-ray Computed Tomography (CT) has become one of the most important diagnostic tools in medicine. After the $\mathrm{X}$-ray attenuation, coefficients through a cross-section of an object are measured in different directions and the distribution of the X-ray attenuation in this cross-section is calculated by a computer. It will help doctors to detect a focused, position of a human body. The attenuation coefficients of the focus are always different from the normal conditions. On account of metal object's attenuation to the diagnostic X-rays is much greater than soft tissues ,bones, and so on, so fewer photons can reach detectors. Before scanning, patients need to take off the metal objects, but many metal objects are difficult to remove such as dental fillings, hip prostheses, these metals produce metal artifacts. It is well known that when the filtered back-projection (FBP) method is used to reconstruct images, the projections must be complete ${ }^{[1]}$. But when images have metals, pronounced dark and bright streaks are produced in the reconstruction. These artifacts seriously degrade image quality. Especially near metal areas, this problem makes diagnosis very difficult.

Considering the basic principle of $\mathrm{CT}$ reconstruction images, there are two methods to remove the undesirable affects within the metal artifact field. The first method is based on the view point of projection compensation. Some projections of metal object could be distorted or missed. Before reconstruction, compensation for the missing data is needed. And the method of projection compensation is based on linear interpolation. The second method is based on the view point of reconstruction method. The iterative reconstruction methods include the algebraic reconstruction technique (ART) ${ }^{[2]}$ and the other two approaches-MLEM ${ }^{[2]}$ and OSEM ${ }^{[3]}$ based on statistical iteration. During the projection compensation process in metal area, the first step is the image segmentation to discover the metal area, and then conduct projection again in this area. To this range of projection data, we will use interpolation to compensate the projection data. Generally, there are three medical image segmentation methods: (1) threshold segmentation method, (2) edge segmentation method, (3) region segmentation $\operatorname{method}^{[4]}$.

In this paper, we are interested in segmentation of metal and normal tissue. We introduces principle of CT image reconstruction in section 2.Then, we mainly discusses the threshold segmentation in section 3 . 


\section{The principle of $\mathbf{C T}$ image reconstruction}

\subsection{Mathematical description}

Assuming the input X-ray is monoenergetic photon, it passes through a homogeneous material. We measure X-ray intensity on both sides of the incidence and emergence. As Lambert Beer law gives following formula-:

$$
I=I_{0} e^{-\mu_{0} \Delta x}
$$

where $I_{0}$ and $I$ are incident intensity and emergent intensity of the ray respectively; $\Delta x$ is the thickness of the material, $\mu_{n}(n=1,2, \ldots N)$ is the linear attenuation coefficient of the materials. The larger $\mu$ value, the more the X-ray's attenuation. If an object composed of $N$ kinds of materials which have different attenuation coefficients $\mu_{n}$ ( $n=$ $1,2, \ldots N)$,we consider Eq.(1) can be changed as the following formula for the object.

$I=I_{0} e^{-\mu_{1} \Delta x} e^{-\mu_{2} \Delta x} e^{-\mu_{3} \Delta x} \cdots e^{-\mu_{n} \Delta x}=I_{0} e^{\sum_{n=1}^{N}-\mu_{n} \Delta x}$

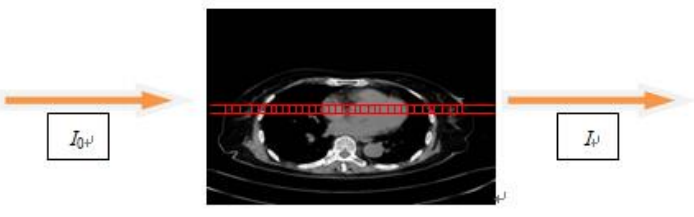

Fig.1 Lambert Beer law-

The transmissivity of the object is expressed in terms of absorbance which is defined as $p$.

$$
p=-\ln \left(\frac{I}{I o}\right)=\sum_{n=1}^{N} \mu_{n} \Delta x
$$

When $\Delta x$ closes to zero, $p$ can be calculated with following integral.

$$
p=\int_{L} \mu_{n} \Delta x
$$

In CT, $p$ is the measured value of the projection. CT reconstruction problems can be regarded as a given line integral of the object.

\subsection{CT image reconstruction steps}

Step1: Based on the original projection data, reconstruct the original image which contains metal artifacts through the filter back projection algorithm.

Step 2: In the original image segmentation out of the metal area, and perform the re-projection to get the scope of the metal area in the range of the projection.

Step 3: Use linear interpolation method in that range of projection data, to get the projection data after compensation.

Step 4: Make use of filter back projection algorithm based on the projection data of interpolation, and get to CT images after reconstruction.

Step 5: Insert the metal part image into the reconstruction of image ${ }^{[5,6]}$

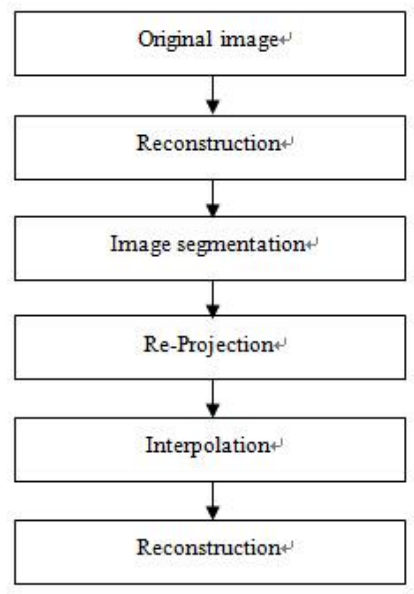

Fig.2 Algorithm flow chart for CT image reconstruction

\section{Medical image segmentation}

\subsection{Mathematical description}

Medical image segmentation is based on a certain similarity characteristics of medical image which could be divided into a number of regions. In the same region, the relevant characteristics show the consistency or similarity. But in different regions can show obvious differences. Generally speaking, there is at least a region of interest (ROI) in a meaningful image segmentation result. Medical image segmentation can be used with set theory model. An image $I$ is considered a union of the above regions.

$$
\begin{gathered}
\bigcup_{j=1}^{N} R_{j}=I \\
R_{j} \cap R_{k}=\phi, \forall j \neq k, j, k \in[1, N]
\end{gathered}
$$

Where $R_{j}$ is the $j$ th region whose pixel satisfy all similarity constraint conditions. $N$ is the number of areas after segmentation $^{[7]}$.

\subsection{Methods of Edge segmentation}

(1)Roberts Operator: The edge location precision is 
relatively high by using a local difference operator to search for the edge, but it is easy to lose a part of edges, meanwhile, it doesn't have the ability to suppress noise for the image smoothing hasn't been done.

(2)Sobel and Prewitt Operators: Sobel and Prewitt operators are to carry out the weighted smoothness to the image firstly, followed by the differential operation, but part of the smooth weight values is different, therefore, they have certain ability to suppress noise, however, they cannot completely eliminate false edges existing in the detection results.

(3)Laplacian Operator: This algorithm is pretty sensitive to the noise, which enhances the noise component, but easy to lose part of the edge directional information, causing not only the discontinuous edge detection but also poorer capability of noise immunity.

(4) Canny Operator: Although it is an edge detection operator derived based on ideas of optimization, the actual effect is not necessarily the most optimal. The reason is that there are always many inconsistencies between theory and practice. This operator adopts Gaussian function to do the image smoothing, therefore, it has better ability to suppress noise, but in the meanwhile, it will also smooth all high-frequency edges into a flat, causing edge losing.

\subsection{Simply threshold segmentation method}

Threshold segmentation is a common method for separation of object and background in an image. The method is essentially based on the statistical characteristics of one dimension gray-level histogram method ${ }^{[8]}$.It is simple to use one or several threshold image gray histogram divided into two or more paragraphs, and the image gray values in the same period of all the pixels belonging to the same object. The traditional threshold segmentation method usually uses the one dimension gray histogram to determine one or more threshold. Ideally, according to the gray-level histogram, direct selection of the appropriate threshold can be very effective in distinguishing different types of organizations (such as the CT image of the skin, bones and other hard or soft tissue), but in most cases, a simple threshold method can't get correct segmentation. Especially, the image contains multiple metal elements. The segmentation in Fig. 3 of $\mathrm{T}=110$ doesn't have reasonable threshold values, so it falsely considers some pixels as the goal region as the goal region, or loses some target pixels. $\mathrm{T}=180$ has better results, but some targets are miss. The reason for this is that a one-dimensional histogram of the image is the occurrence frequency of each pixel gray value in an image, but it does not reflect a grey value of pixels corresponding to the position and the characteristics of the neighborhood. It ignores the spatial neighborhood information. In the gray-level histogram, there may be no significant statistical characteristics. How to determine the optimal threshold segmentation is still a challenging problem at the current stage.

\subsection{Iterative method for optimal threshold segmentation}

Iterative method is based on the optimal approximation and iterative process to choose a best threshold to realize image segmentation. The basic algorithm is as follows: Statistics of the maximum and the minimum of each pixel in the image: $G \max$ and $G \min$. Iterative control variables is $k$, and the threshold value is determined by

$$
T_{k}=\left(G_{\max }+G_{\min }\right) / 2
$$

When $k=0$, it looks as the initial threshold, According to $T_{k}$, make image segmentation of target and background region. The average grey value of the two regions is $M_{0}$ and $M_{1}$. Make the iterative control variables $k=k+1$, to get the new iteration threshold $T_{\mathrm{k}+1}$.
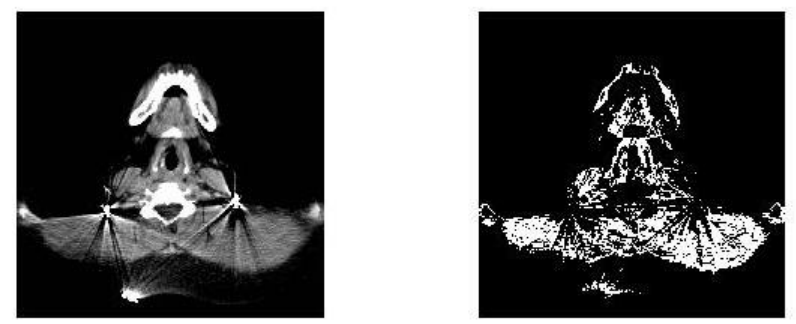

(a) $\mathrm{T}=100$
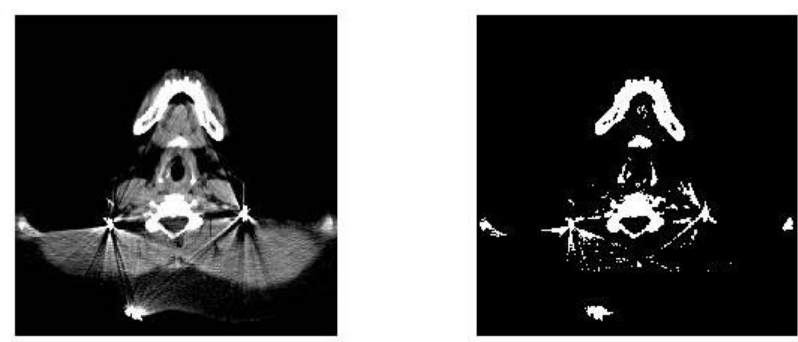

(b) $\mathrm{T}=180$

Fig.3 Simply threshold segmentation method 

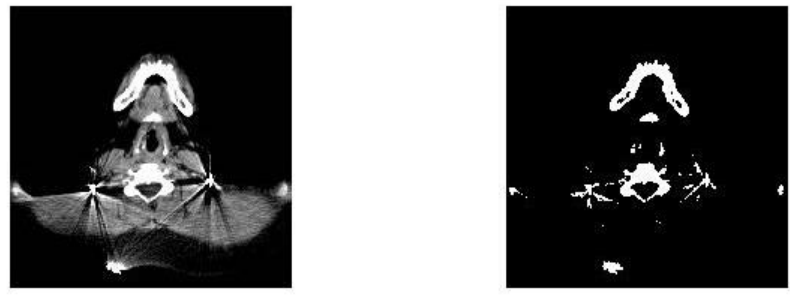

Fig.4 Iterative optimal threshold method

\section{Conclusions}

Human beings have developed a variety of medical image segmentation methods to solve the problem of metal artifacts. How to make use of the various methods has become a major issue in this research field. Different methods of medical image segmentation show a large degree of difference in performance. The development trend highlights accurate, rapid, robustness and adaptive segmentation ${ }^{[9]}$. Because of the complexity of metal images, the general method cannot meet the requirement for practical use and new theories and techniques are needed, Researches are facing many challenges. There is still a long way to go for the wide spreading application of new technologies in clinics. With the continuous development of computer technology and the application and improvement of various new theories, as well as medical image technology, we believe that the medical image segmentation technology will constantly mature ${ }^{[10]}$.as new theories are integrated into the field.

\section{Acknowledgment}

This paper is supported by following fund projects: (1) Jiangsu Province 7th Projects for "Summit Talents in Six Main Industries"; Electronic Information industry, DZXX-149, No.110). (2) Suqian science and technology innovation fund project, No. Z201206. (3) National Natural Science Foundation of China (NSFC, No.61301220).

\section{References}

(1) Dan Xia, John C.Roeske, Lifeng Yu et al: “A hybrid approach to reducing computed tomography metal artifacts in intracavitary brachytherapy”,Brachytherapy, Vol.4(1),pp.18-23, 2005

(2) Hsieh J:"Adaptive streak artifact reduction in computed tomography resulting from excessive X-ray photon noise," Journal of Medical Physics. Vol.25,No.11,pp.2139-2147,1998

(3) Li Zhipeng, Cong Peng, Wu Haifeng:"Research of ART method in CT image reconstruction," Journal of nuclear electronics and detection technology, Vol.25,No.2 pp.184-186,2005

(4) Qiu Ming, hang Erhu: "Methods of medical-image segmentation", Journal of Computer Engineering and Design,Vol.26(6),No.6,pp.1557-1588,2005

(5) Karimi S, Cosman P, Wald C, Martz H: "Segmetation of artifacts and anatomy in CT metal artifact reduction”,MED Phys;39(10):5857-68. doi: 10.1118/1.4749931,2012

(6) Shiying Zhao, Douglas D.Robertson, Ge Wang et al: "X-Ray CT metal artifact reduction using wavelets: An application for imaging total hip prostheses, IEEE Transactions on Medical Imaging ", Vol.19, No.20, 1238 1247,2000

(7) Lin Yao,Tian Jie: "A Survey on medical image segmentation methods", Journal of Pattern Recognition and Artificial Intelligence, Vol.15, No.2,pp.193-204,2002

(8) Sahoo PK,Soltani S,Wang A K C,Chen Y C A : "Survey of Thresholding Techniques Computer Vision", Journal of Graphics and Image Processing, Vol.41, Nov.2 pp.233-260, 1998

(9) Weng Xuan,Zheng Xiaolin, Peng Chenglin: "Research on Medical Image Segmentation Technology", Journal of China medical equipment information, Vol.12, No.6, pp. 24-26,2006

(10) H.S.Prasantha, Shachidhara.H.L,K.N.B.Murthy: "Medical Image Segmentation", IJCSE, Volume, 2(4): 1209-1218,2010 